\title{
Audiovisual processing of pharyngealization and length in Emirati Arabic
}

\author{
Mark Scott ${ }^{1}$, Ali Idrissi ${ }^{2}$ \\ ${ }^{1}$ United Arab Emirates University, Al Ain, Abu Dhabi, UAE \\ ${ }^{2}$ Qatar University, Doha, Qatar \\ https://doi.org/10.36505/ExLing-2015/06/0019/000256
}

\begin{abstract}
This paper addresses a gap in the literature on audiovisual speech perception. Previous research has examined how the perceived primary place-of-articulation of a speech sound is influenced by visual information. Visual influences on the perceived length of a speech sound or on the presence of pharyngealization have not been examined. The experiments reported here demonstrate that the perception of both singleton/geminate and pharyngealized/plain is susceptible to visual influence.
\end{abstract}

Key words: Audiovisual, perception, geminate, pharyngealization, Arabic

\section{Introduction}

Most studies of the McGurk effect have demonstrated that the perceived placeof-articulation of a sound can be shifted by visual information. Arabic has contrasts that are not so clearly about(primary) place-of-articulation. Geminates involve holding a constriction for a longer time; pharyngealization involves a secondary constriction which is not itself visible. The two experiments below test whether Arabic speakers are visually sensitive to these contrasts. The prediction in Experiment One is that the consonant length in a video will shift perceived auditory consonant length to match. In Experiment Two, we predict that video of pharyngealized or plain sounds will shift auditory perception of pharyngealization to match -here the perceiver is not seeing the pharyngeal gesture, but its visual effects on surrounding sounds.

\section{Experiment One: Audiovisual Perception of Gemination}

In comparison to hearing, vision is poor at timing perception. This means that when there is a conflict, we generally perceive timing in line with information from hearing(Goldstone \& Lhamon, 1974).This is the modality appropriateness hypothesis (Welch, 1999) and it suggests (contra this experiment) that visual timing influences on hearing are unexpected.

\section{Procedures}

An audio continuum between /abah/ (singleton) and /abbah/ (geminate) was created by morphing between clear endpoints (recordings of a native Emirati Arabic speaker). The same speaker was video recorded and 8 videos each for

ExLing 2015: Proceedings of 6th Tutorial and Research Workshop on Experimental Linguistics, 26-27 June 2015, Athens, Greece 
singleton and geminate (free of any obvious irregularities) were chosen for use. For both singleton and geminate conditions, two interleaved audio staircases were presented with random switching. Each staircase had12 turns. For the first 6 turns of each staircase, the audio was presented alone; after 6 turns, the audio was accompanied by videos of either singleton or geminate. There were 40 native Arabic speaking female participants.

\section{Results \& Discussion}

Each participant's data was fit to a psychophysical curve.

participants' data were unfittable and were eliminated from analysis. A paired ttest comparing the fitted thresholds between "Long" and "Short" was significant $[\mathrm{t}=-3.1105, \mathrm{df}=32, \mathrm{p}=0.0039]$. This demonstrates that people can integrate audiovisual speech timing information. This is surprising, as the auditory system typically dominates the audiovisual perception of timing.

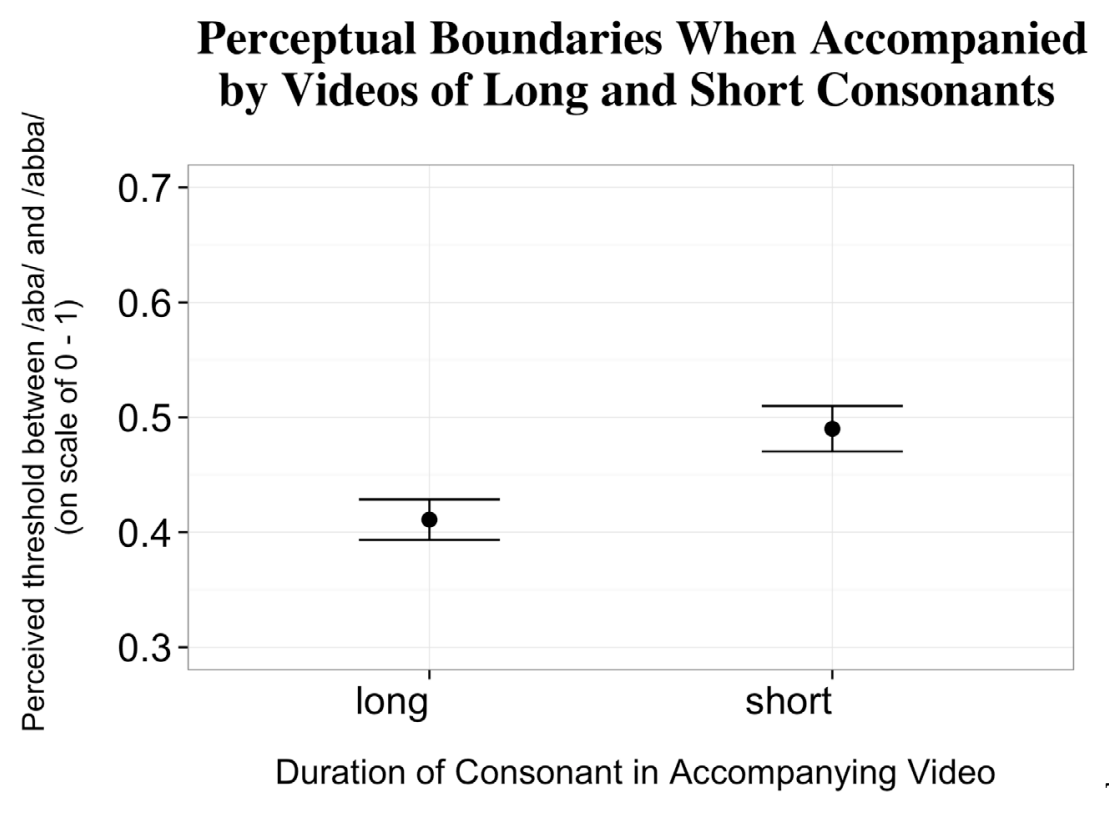

Figure 1. Results of Experiment One - Standard-Error bars are shown. 


\section{Experiment Two: Pharyngealization}

Most Arabic dialects have pharyngealized consonants. This is a secondary articulation that involves a constriction at the upper part of the pharynx (Ladefoged \& Johnson, 2011). Pharyngealization typically spreads to neighbouring segments (Zawaydah, 1999). In Gulf Arabic, this pharyngealization is accompanied by some degree of lip rounding (Watson, 1999).

\section{Procedures}

This experiment was identical in structure to Experiment One except that the sounds were /aðah/ (plain) and /a $\partial^{\varsigma}$ ah/ (pharyngealized). The audio continua and videos were created in the same way as in Experiment One. The same participants as in Experiment One were run in this experiment.

\section{Results \& Discussion}

Each participant's data was fit to a psychophysical curve. 15 participants' data were unfittable and were eliminated from analysis. A paired t-test comparing the fitted thresholds between "Pharyngealized" and "Plain" was significant [ $\mathrm{t}=$ -2.4992, df $=24, \mathrm{p}=0.0197]$. As predicted, more pharyngeal were heard with a pharyngeal video and similarly for plain.

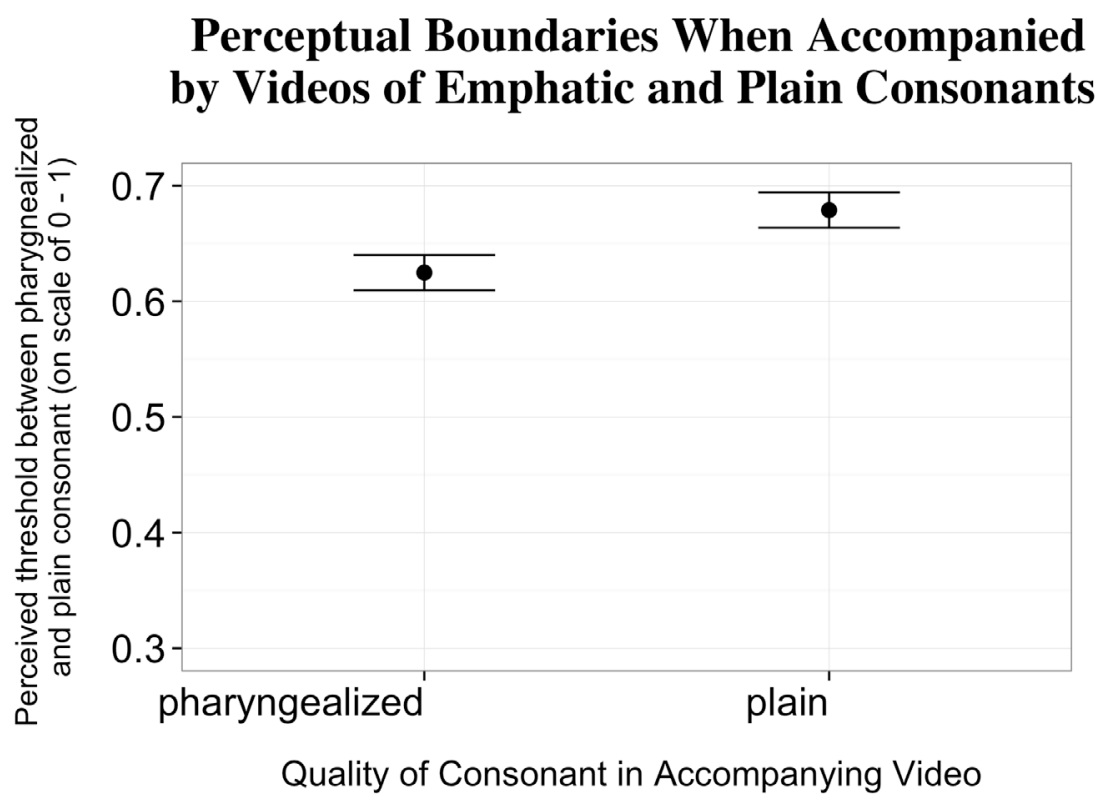

Figure 2. Results of Experiment Two - Standard-Error bars are shown. 
This shows that Arabic speakers are sensitive to visual cues to pharyngealization, and can integrate these cues into a combined audiovisual percept. Clearly, participants are not seeing the secondary constriction at the pharynx itself, as this is necessarily occluded. Instead, pharyngealization is being detected via other visual cues such as jaw-retraction and lip rounding.

\section{Conclusions}

The use of visual cues to length and secondary articulations has not received much attention. These experiments address this gap by showing that visual information about the duration (singleton versus geminate) of a consonant and the presence of a secondary articulation (pharyngealization) on segments can be detected visually and integrated into an audiovisual percept.

\section{Acknowledgements}

This research was supported by UAEU Grant 31h076. We would like to thank our Assistants: Souad Al Helou and Hamdha Saleh Salem Alkarbi.

\section{Referencess}

Goldstone, S. and Lhamon, W.T. 1974. Studies of auditory-visual differences in human timing judgment: Sounds are judged longer than lights. Perceptual and Motor Skills (39), 63-82.

Ladefoged, P. and Johnson, K. 2011. A Course in Phonetics 6th ed., Cengage Learning, Wadsworth.

Watson, J.C.E. 1999. The Directionality of Emphasis Spread in Arabic. Linguistic Inquiry 30(2), 289-300.

Welch, R.B. 1999. Chapter 15 Meaning, attention, and the 'unity assumption' in the intersensory bias of spatial and temporal perceptions. In Aschersleben, G., Müsseler, J. \& Bachmann, T. (eds.), Advances in Psychology, 371-387, New York, North-Holland.

Zawaydah, B.A. 1999. The phonetics and phonology of gutturals in Arabic. Ph.D. Indiana: Indiana University. 